\title{
Critical and distinct roles for key RET tyrosine docking sites in renal development
}

\author{
Sanjay Jain, ${ }^{1,5}$ Mario Encinas, ${ }^{2,4}$ Eugene M. Johnson Jr., ${ }^{2}$ and Jeffrey Milbrandt ${ }^{3,6}$ \\ ${ }^{1}$ Department of Surgery, ${ }^{2}$ Department of Molecular Biology and Pharmacology, ${ }^{3}$ HOPE Center for Neurological Disorders, \\ Department of Pathology, and Department of Neurology, Washington University School of Medicine, St. Louis, Missouri \\ 63110, USA; ${ }^{4}$ de Ciencies Mediques Basiques, Laboratori d'Investigacio, HUAV/UdL, Lleida 25198, Spain
}

\begin{abstract}
Molecular mechanisms that lead to congenital anomalies of kidneys and the lower urinary tract (CAKUT) are poorly understood. To elucidate the molecular basis for signaling specificity of GDNF-mediated RET signaling in kidney development, we characterized mice that exclusively express either the human RET9 or RET51 isoform, or express these isoforms with individual mutations in docking tyrosines for PTB and SH2-domain-containing adaptors Src (Y981), PLC $\gamma$ (Y1015), and She (Y1062). Our results provide evidence for differential and isoform-specific roles of these docking sites in murine kidney development. Homozygous $\operatorname{Ret}^{R E T 9}$ and $\operatorname{Ret}^{R E T 51}$ mice were viable and show normally developed kidneys, indicating redundant roles of human RET isoforms in murine kidney development. In the context of the RET51 isoform, only mutation of the docking Tyr 1015 (Y1015F) resulted in severe renal anomalies. These included bilateral megaureters and multicystic kidneys that were caused by supernumerary ureteric buds that fail to separate from the wolffian duct as well as decreased branching morphogenesis. Similar kidney and ureter defects were observed in RET9(Y1015F) mice that contain the Y1015F mutation in the RET9 isoform. Interestingly, loss of RET9(Y1062)-mediated AKT/MAPK activation resulted in renal agenesis or kidney rudiments, whereas mutation of this residue in RET51 had no obvious effect on AKT/MAPK activity and renal development. These results reveal novel roles of key RET-dependent signaling pathways in embryonic kidney development and provide murine models and new insights into the molecular basis for CAKUT.
\end{abstract}

[Keywords: RET; GDNF; kidney; RTK; CAKUT]

Supplemental material is available at http://www.genesdev.org.

Received October 20, 2005; revised version accepted December 14, 2005.

Several biological processes such as organogenesis and diseases like cancer depend on receptor tyrosine kinase (RTK) signaling. Upon activation by ligands/coreceptors, RTKs typically interact with intracellular adaptor proteins via docking phosphotyrosines and activate several signaling cascades such as PKC, AKT, and MAPK (Madhani 2001). While physiological roles of several RTKs have been elucidated by gene knockout studies, little is known about how specific intracellular signaling pathways meditate their pleiotropic effects in vivo.

The RET RTK is critical for the development of the lineages of the peripheral nervous system, the kidneys, and the testes (Sariola and Saarma 2003; Jain et al. 2004). Congenital renal abnormalities are one of the most common developmental defects in children. Aberrant RET

Corresponding authors.

${ }^{5}$ E-MAIL jainsa@msnotes.wustl.edu; FAX (314) 362-8756.

${ }^{6}$ E-MAIL jeff@pathbox.wustl.edu; FAX (314) 362-8756.

Article and publication are at http://www.genesdev.org/cgi/doi/10.1101/ gad.1387206. signaling in the developing murine kidney results in congenital anomalies of the kidneys and the lower urinary tract (CAKUT) (Pope et al. 1999). These include renal agenesis, hypoplasia, hypodysplasia, multicystic kidneys, and defective ureteral maturation (Schuchardt et al. 1994; Srinivas et al. 1999; de Graaff et al. 2001; Enomoto et al. 2001; Batourina et al. 2002; Jain et al. 2004; Jijiwa et al. 2004). Recent evidence also suggests that genitourinary (GU) defects due to deficiencies in other signaling proteins may also be mediated via RET (Grieshammer et al. 2004; Basson et al. 2005), but the molecular basis for how deficits in RET signaling lead to such a wide array of GU defects is unknown.

RET forms a signaling complex with one of the GDNF family ligands (GFLs-GDNF, Neurtirin, Artemin, or Persephin) and a coreceptor GFR $\alpha(\mathrm{GFR} \alpha 1-4)$ (Baloh et al. 2000). In renal development, GDNF-mediated RET signaling is physiologically important as Gdnf-, Gfro1-, or Ret-null mice die perinatally due to bilateral renal agenesis (Schuchardt et al. 1994, 1996; Moore et al. 1996; Pichel et al. 1996; Sanchez et al. 1996; Cacalano et al. 
1998; Enomoto et al. 1998, 2001). Kidney development depends on reciprocal inductive interactions between the GDNF-producing mesenchyme and the ureteric bud (UB) epithelium, which expresses RET/GFR $\alpha 1$ (Lechner and Dressler 1997; Yu et al. 2004). At embryonic day 11.5 (E11.5), GDNF initiates UB outgrowth from the wolffian duct, which, in turn, invades the metanephric mesenchyme, causing its differentiation into epithelium and the formation of nephrons. In turn, the mesenchyme secretes factors that promote branching morphogenesis of the UB that eventually differentiates into the collecting duct system. Disruption of any of these events results in CAKUT.

RET activation results in phosphorylation of key docking tyrosines that bind to several adaptor proteins such as Src (at Y981); PLC $\gamma$ (at Y1015); Shc; FRS2; IRS1 and IRS2; Enigma; Doks 4, 5, and 6 (at Y1062); and Grb2 (at Y1096). These interactions activate downstream signal transduction pathways such as Raf/Ras (Src), PKC (PLC $\gamma$ ), PI3K/AKT, and MAPK (Shc and Grb2) (Airaksinen and Saarma 2002). These cascades mediate RETstimulated cell proliferation, migration, differentiation, and survival. RET signaling via specific intracellular signaling cascades may be regulated by alternative splicing, as there are two major splice isoforms, RET9 and RET51. RET9 (1072 amino acids long) and RET51 (1114 amino acids long) diverge after residue 1063, and thus are identical except for the C-terminal cytoplasmic domain (Takahashi 2001). The physiologic roles of these two isoforms and the signaling pathways emanating from specific phosphorylated tyrosine docking sites have been studied in vitro, but information in vivo is limited. The relative importance of the RET isoforms and particular docking sites has differed depending on the system investigated (Liu et al. 1996; Rossel et al. 1997; Borrello et al. 2002; Tsui-Pierchala et al. 2002). In mice that exclusively express chimeric mouse-human Ret9 or Ret51, it was found that Ret9 alone is sufficient for kidney development, whereas Ret51 did not promote normal renal formation (de Graaff et al. 2001).

To determine if specific RET phosphotyrosine docking sites guide distinct biological processes, and to study the molecular basis of RET-mediated pleiotropic effects in kidney development and CAKUT, we characterized mice that harbor individual RET(Tyr-Phe) mutations in the human RET9 and RET51 isoforms. In contrast to a prior report, we find that both homozygous $\operatorname{Ret}^{R E T 9}$ and Ret $^{\text {RET51 }}$ kidneys develop normally (de Graaff et al. 2001). However, individual phosphotyrosine docking sites of these isoforms have specific roles in kidney development. The disruption of Y1015-mediated PLC $\gamma$ activation, in either the RET9 or RET51 isoform, results in supernumerary UBs and hypodysplastic kidneys with decreased branching morphogenesis and increased apoptosis, leading to changes consistent with obstructive nephropathy and death by 1 mo of age. Interestingly, the Y1062F mutation led to severe renal dysgenesis, similar to that seen in Ret-null mice, in RET9, but not in RET51 mice. These results indicate that the docking tyrosines of the two RET isoforms have different signaling properties in vivo. These genetically defined RET mutant mice provide useful models to study the congenital defects of the GU system and will help define the molecular mechanisms underlying these defects.

\section{Results}

Generation of mice expressing RET signaling mutants

To study the roles of RET isoforms and their key docking tyrosines (Y981, Y1015, Y1062) in kidney development, we targeted wild-type human RET9 or RET51 cDNA, or the indicated mutant cDNAs of each of these isoforms into the endogenous Ret locus using homologous recombination (knockout-knockin approach) (Fig. 1A) as previously described (Enomoto et al. 2001; Jain et al. 2004). This strategy ensured that we could properly compare phenotypes between mice harboring RET mutations with those expressing the wild-type knocked-in RET isoforms. A total of 10 different genetically engineered RET mutant mice were created (Fig. 1B). We used RT-PCR analysis of brain RNA to detect mRNAs corresponding to endogenous mouse Ret, the isoform-specific RET9 or RET51, and total RET to confirm the successful generation of each respective RET mutant, and to show that homozygous mutant mice had no endogenous mouse Ret expression (Fig. 1C). Immunohistochemical analysis confirmed that RET protein was detected in the nephrogenic zone of the kidneys or in other Ret-dependent target tissues in all RET mutant mice (Fig. 1D; data not shown). All of the homozygous RET mutant mice were born at the expected Mendelian frequencies as anticipated since RET is not required for survival during embryogenesis (Schuchardt et al. 1994; Enomoto et al. 2001). For all of the studies reported here, RET mutant mice from a mixed genetic background (129/SvJ:C57BL/ 6: F2-F5) were used.

\section{RET isoforms have redundant roles in kidney formation}

We generated Ret ${ }^{\text {RET9/RET9 }}$ and $R e t^{\text {RET51/RET51 mice to }}$ examine the biological differences between the human RET isoforms and to use these monoisomorphic mice (exclusively express one RET isoform) as controls for mice expressing each of the respective RET monoisomorphic mutants. In contrast to previous reports, we found that both $\operatorname{Ret}^{\text {RET9/RET9 }}$ and $\operatorname{Ret}^{\text {RET51/RET51 mice }}$ were viable. We performed biochemical analysis using superior cervical ganglion (SCG) neurons from each of these mice and found that the expression of RET in these monoisomorphic mice was comparable $(-60 \%-80 \%$ of wild-type Ret levels), and that GDNF-dependent activation of MAPK and AKT was intact in both $\operatorname{Ret}^{\text {RET9/RETP }}$ and $\operatorname{Ret}^{R E T 51 / R E T 51}$ mice (Fig. 2A). Further analysis of these mice revealed normally located gonads, no overt defects in the enteric nervous system, and essentially normally developed kidneys $(n>50$ mice for each genotype) (Fig. 2B; data not shown). The only urinary system 
A

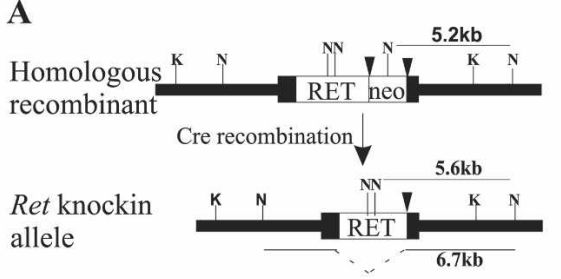

B

RET9 or RET51

RET51(Cdel)

$\operatorname{RET9(51C)}$
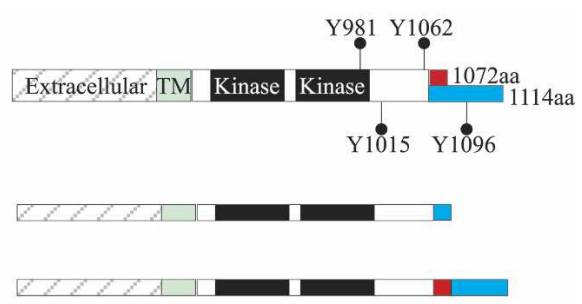

C

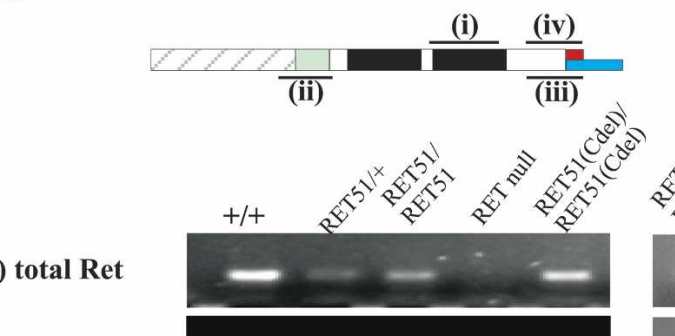

(ii) mouse Ret

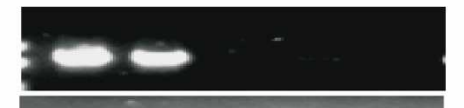

(iii) total Ret51

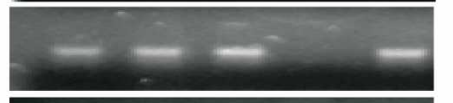

(iv) total Ret9
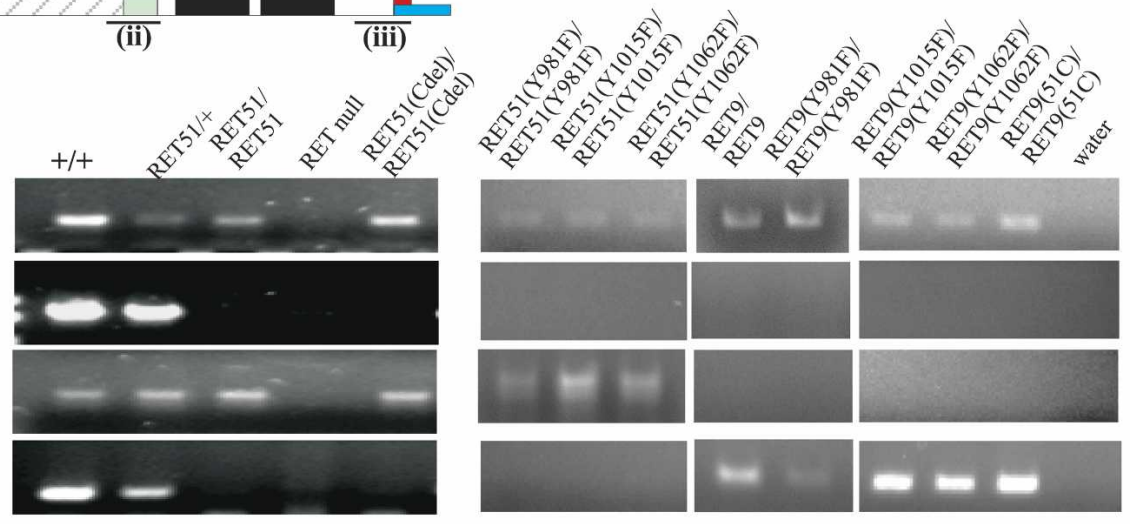

D
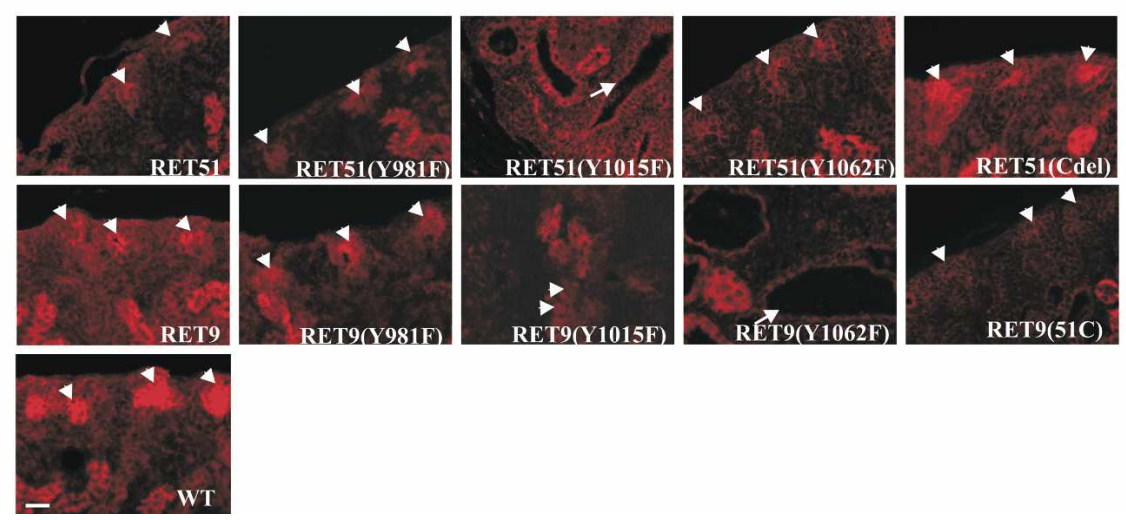

Figure 1. Generation of mice expressing various RET signaling mutants. (A) Mutant or wild-type human RET cDNAs were introduced into exon 1 (black box) of the mouse Ret locus (black triangles, loxP sites). The restriction sites ([K] KpnI; [N] NcoI) and length of the expected fragments in Southern blots are indicated. (B) Schematic of the different wild-type RET9, RET51, and their respective mutant knocked-in alleles. The different domains and sizes of the RET9 and RET51 alleles are indicated. The red and blue colors represent the area of divergence between the two RET isoforms. Also indicated are the key docking tyrosine $(\mathrm{Y})$ residues. Homozygous mice were generated that harbor Tyr $\rightarrow$ Phe (Y $\rightarrow$ F) for each of the indicated tyrosines except Y1096. RET51(Cdel) is an allele in which residues 1063-1072 of RET9 were replaced with residues 1063-1072 of RET51 (results in receptor that is essentially similar to RET51 with deletion of residues 1073-1114, including Y1096). RET9(51C) is an allele in which residues 1063-1072 of RET51 were replaced with residues 1063-1072 of RET9 (results in a receptor that is essentially similar to RET9 with the C terminus of RET51 including Y1096). (C, top) Schematic depicting the position of amplicons representing various mRNAs (indicated by numbers) of RET isoforms. (Bottom) RT-PCR using total brain RNA depicts the successful generation of each mutant homozygous monoisomorphic RET mouse. Labeling on the left indicates the RET allele being detected: (total RET) detects mouse and/or human RET; (mouse Ret) detects endogenous mouse Ret; (total RET51) detects mouse and/or human RET51 isoform; (total RET9) detects mouse and/or human RET9 isoform. Labeling on the top indicates the genotype at the Ret locus: $(+)$ endogenous mouse allele; $(-)$ null allele. $(D)$ RET immunohistochemistry on P0 kidneys depicts RET expression in the nephrogenic zone (arrowheads) of the indicated homozygous mutant animals. The abnormal disorganized renal architecture in mice expressing RET51(Y1015F), RET9(Y1015F), and RET9(Y1062F) is due to renal abnormalities; see later (arrows, kidney tubules). Bar, $25 \mu \mathrm{m}$. 
Jain et al.

A

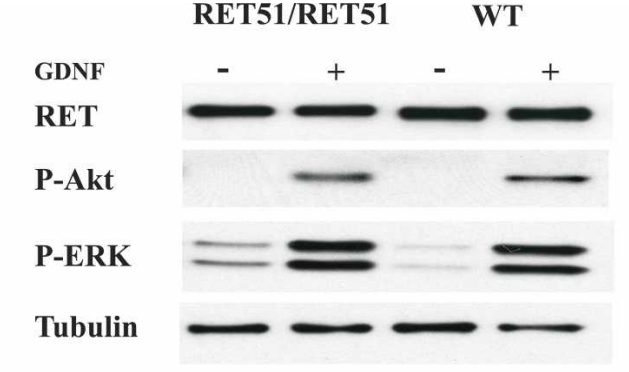

B

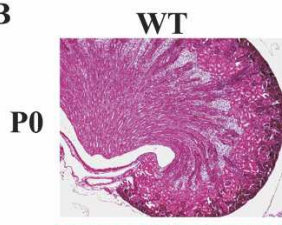

$2 \mathrm{~m}$

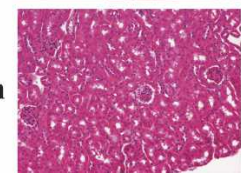

RET51

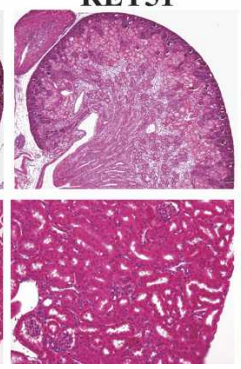

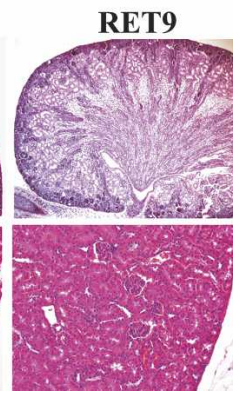

RET9/RET9 WT
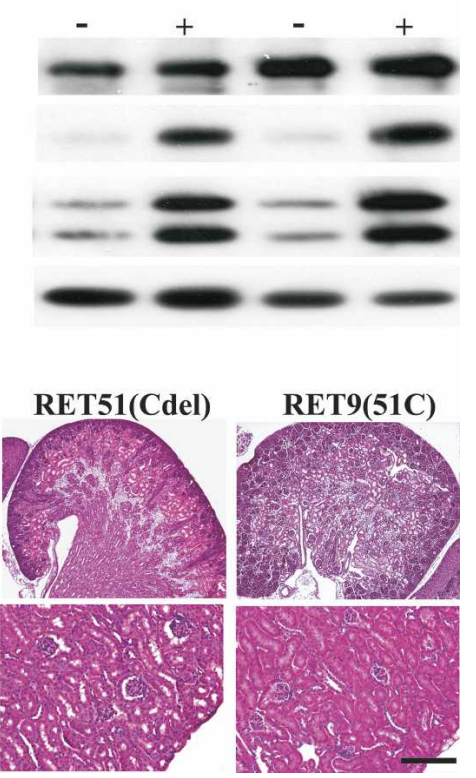

RET9(51C)

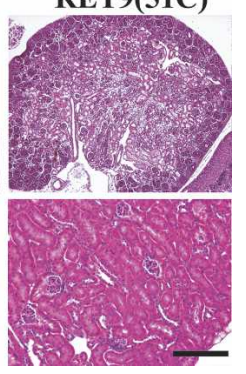

Figure 2. Mice expressing either RET51 or RET9 show normal renal development and activation of AKT/MAPK pathways. (A) Immunoblots of extracts from primary SCG neurons (cultured for $8 \mathrm{~d}$ ) show similar GDNF-dependent phosphorylation of downstream signaling effectors in homozygous RET51, RET9, and wild-type mice (WT). The antibodies used were Ret (total RET), P-Akt (phospho Akt), and P-ERK (phospho ERK). The blot was reprobed with tubulin antibody to ensure equal loading. (B) H\&E-stained kidney sections of homozygous mice for the indicated knocked-in alleles at P0 and $2 \mathrm{mo}(2 \mathrm{~m})$ of age show normal kidney histology. Bar: P0, $200 \mu \mathrm{m}$; $2 \mathrm{~m}, 50 \mu \mathrm{m}$.

defect in these mice that we have not observed in wildtype mice was a low penetrance unilateral proximal ureter dilatation/hydronephrosis in homozygous RET51 $(1 / 50)$ and RET9 (3/50) mice. The Ret RET9/RET9 and Ret $^{\text {RET51/RET51 }}$ mice we generated live past 1 yr of age.

It was previously postulated that alternative physiological roles of these two RET isoforms could be due to differences in their C termini (Liu et al. 1996; Rossel et al. 1997; de Graaff et al. 2001). To examine this hypothesis further, we generated two additional RET mutant mice. In these mutants the nine residues after RET1063 were swapped between RET9 and RET51 cDNAs [RET9(51C) and RET51(Cdel)] to create chimeric RET isoforms (Fig. 1B). Surprisingly, we found that $\operatorname{Ret}^{\text {RET9(51C)/RET9(51C) }}$ and Ret RET51(Cdel)/RET51(Cdel) mice were also viable and had normally developed kidneys (Fig. 2B). From these observations, we conclude that wild-type human RET9 and RET51 isoforms have redundant roles and they can execute normal kidney development in the absence of mouse Ret. Furthermore, the C terminus of RET51 encompassing the extra docking tyrosine Y1096 for Grb2 adaptor (not present in RET9) is dispensable in vivo.

\section{RET51(Y1015) docking tyrosine is essential for proper formation of kidneys, ureters, and gonads}

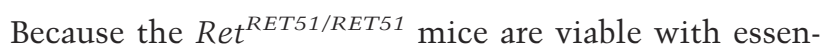
tially normal kidneys, we therefore investigated if the docking tyrosines Y981, Y1015, and Y1062 are important for kidney development in homozygous $\operatorname{Ret}^{\text {RET51(Y981F), }}$ $\operatorname{Ret}^{\text {RET51(Y1O15F) }}$, and Ret $t^{\text {RET51(Y1062F) }}$ mice. We observed that all $\operatorname{Ret}^{\text {RET51(Y1O15F)/RET51(Y1O15F) }}$ mice died by $5 \mathrm{wk}$ of age. The inability of Ret RET51(Y1O15F)/RET51(Y1O15F) mice to survive to adulthood is likely due to severe bilateral GU defects (Fig. 3). Postnatal analyses of kidneys

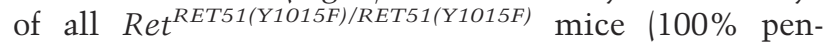
etrance, $n>10$ ) show severe dilatation of both ureters (megaureters), hydronephrosis, obliterated renal parenchyma with multiple cysts, and histopathological changes consistent with obstructive nephropathy (Fig. 3A).

To determine if these defects occurred postnatally or were congenital, we examined Ret ${ }^{\text {RET51(Y1015F)/RET51(Y1O15F) }}$ kidneys at birth (P0, $n>15)$. These kidneys exhibit a subset of abnormalities present in human CAKUT (Pope et al. 1999), including bilateral hypodysplasia, multiple cysts, and bilateral megaureters with distal stenosis (Fig. 3B). Interestingly, both gonads (ovaries and testes) failed to descend to their normal locations due to the inability of the vas deferens/wolffian duct to separate from the ureters (Figs. 3B, 4B; S. Jain, unpubl.). Consistent with hypoplasia, there was a severe reduction in the number of glomeruli in Ret ${ }^{\text {RET51(Y1O15F)/RET51(Y1O15F) kidneys. In }}$ comparison, P0 kidneys of $\operatorname{Ret}^{\text {RET51(Y981F)/RET51(Y981F) }}$ $(n>7)$ and Ret RET51(Y1062F)/RET51(Y1O62F) $(n>30)$ mice were grossly or histologically normal as evidenced by presence of an outer nephrogenic zone, and organization into a defined cortex, medulla, and pelvis (Fig. 3C). Low penetrance unilateral ureteral dilatation was seen in homozygous RET51(Y1062F) mice (1/60). These studies es- 
A
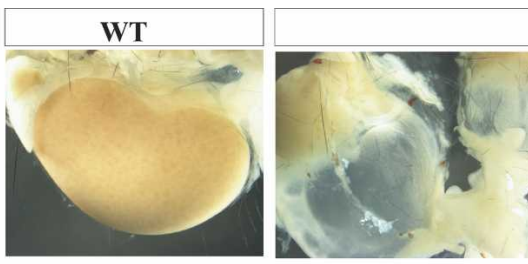

RET51(Y1015F)

B

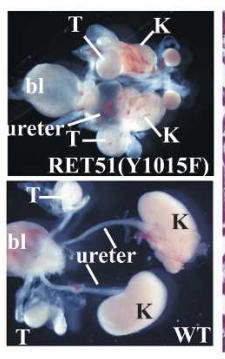

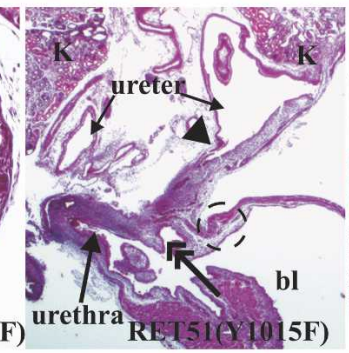

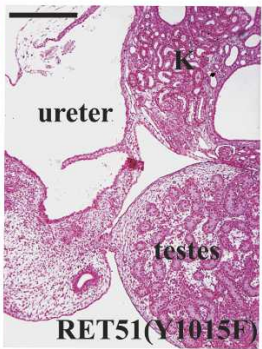

RET51(11015F
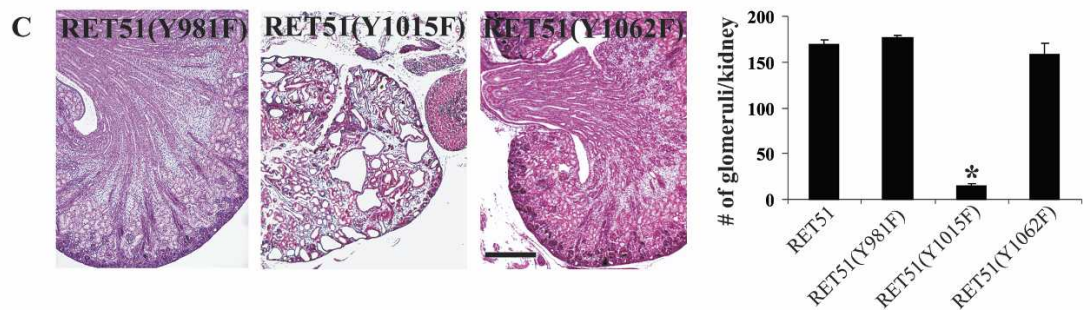

Figure 3. Mice expressing RET51(Y1015F) exhibit severe ureter and kidney abnormalities. (A) Compared with wild-type (WT), kidneys from 5-wk-old homozygous RET51(Y1015F) mice exhibit megaureters and renal atrophy. H\&E sections at low power demonstrate decreased renal parenchyma and dilated ureter (bar, $200 \mu \mathrm{m}$ ). The micrograph at high power on the right shows end-stage renal histology depicted by globally sclerosed glomeruli (g), inflammation (arrow), and multiple cystic tubules (asterisks) with exudates and tubular atrophy (bar, $100 \mu \mathrm{m})$. (B) Renal abnormalities in homozygous RET51(Y1015F) mice are congenital. (Left) Morphology of wild-type (WT) kidneys (K) depicting normal entry of ureters into the bladder (bl). The gonads ([T] testis) and their ducts are separated from the urinary tract and are positioned near the bladder. The GU system of homozygous RET51(Y1015F) mice is composed of small kidneys, megaureter, and testes attached to the kidney/ureter. (Right) The H\&E-stained sections show hypodysplastic kidneys with multiple cysts of varying sizes (first panel, scale bar, $50 \mu \mathrm{m})$, dilated ureter with a distal stricture (arrowhead) that appeared to enter the proximal urethra (double-headed arrow) instead of the bladder (dashed circle) (second panel, scale bar, $800 \mu \mathrm{m}$ ), and testes aberrantly positioned next to the proximal ureter and kidney (third panel, bar, $250 \mu \mathrm{m}) .(C)$ Comparison of the indicated RET mutants at P0 shows that kidneys of only the homozygous RET51(Y1015F) mice are severely abnormal. The graph on the right shows a severe decrease in the number of glomeruli in kidneys of RET51(Y1015F) mice compared with the other RET mutants $\left(n=6\right.$ kidneys, 3 animals of each genotype; $\left.\mathrm{mean}_{ \pm} \mathrm{SEM} ;\left[^{\star}\right] P<0.05\right)$. Bar, $200 \mu \mathrm{m}$.

tablish that in the context of the human RET51 isoform, the docking tyrosine Y1015 is critical for proper development of both ureters, kidneys, and gonads, whereas RET51(Y981), RET51(Y1062), and RET51(Y1096) are largely dispensable.

Supernumerary UBs and decreased branching morphogenesis in the absence of RET51(Y1015) signaling

Defects in the development of the UB have been proposed to cause CAKUT (Pope et al. 1999). In mouse kidney development, UB outgrowth occurs by E11.5, and by E12.5 it has typically invaded the metanephric mesenchyme and undergone initial branching (Vize et al. 2003). By E15.5, the developing kidney is organized into an outer cortex and an inner medulla, the mesenchymal to epithelial transition has been initiated to produce various components of the nephron, and the ureters have separated from the wolffian duct and formed connections to the bladder. To determine the etiology of CAKUT-like defects in Ret ${ }^{\text {RET51(Y1015F)/RET51(Y1015F) }}$ kidneys, we compared the development of these kidneys with that of wild-type (WT) and homozygous RET51 and RET51(Y1062F) kidneys at E12.5. Histological analysis at E12.5 revealed that the UB successfully reached the metanephric mesenchyme and underwent initial branching as demonstrated by the presence of UB derivatives (Fig. 4A). Note also the presence of ectopic foci of the developing kidney in Ret RET51(Y1015F)/RET51(Y1015F) mice, suggesting that these mice have early UB development abnormalities. By E15.5, the abnormalities in $\operatorname{Ret}^{\text {RET51(Y1015F)/RET51(Y1015F) }}$ kidneys were readily evident. Histological analysis clearly highlights the ectopic kidney, suggesting the possibility of multiple UB inductions. Furthermore, the Ret RET51(Y1015F)/RET51(Y1015F) kidneys fail to establish a normal architecture including an outer cortex and inner medulla that normally occurs in wild-type and homozygous RET51 and RET51(Y1062F) animals. Developing glomeruli were identified in

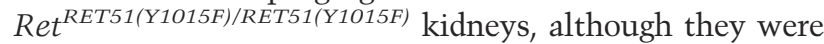
scant and were scattered throughout the mesenchyme. This suggests that the mesenchyme of these mice has the ability to undergo differentiation to produce glomeruli; however, they are reduced in number presumably due to UB branching deficits (Majumdar et al. 2003).

We next performed whole-mount dolichos biflorus agglutinin (DBA) staining on the E15.5 GU system of Ret $^{\text {RET51(Y1015F)/RET51(Y1015F) }}$ mice to visualize the urinary tree for abnormalities in branching morphogenesis and the ureters (Sainio et al. 1997; Grisaru et al. 2001; 
Jain et al.

Figure 4. Characterization of GU defects mediated by RET51(Y1015F) during embryogenesis. (A) H\&E sections of E12.5 and E15.5 kidneys of indicated RET homozygous mutants. A duplicated kidney (K1 and $\mathrm{K} 2)$ is evident in homozygous RET51(Y1015F) animals at E12.5. The lumen represents the initial branching of the UB and indicates that the UB was able to invade the metanephric mesenchyme. Also note the mesenchymal condensation $(\mathrm{mc})$, indicating at least some degree of mesenchymal differentiation in RET51(Y1015F) kidneys. At E15.5, the abnormal architecture of mutant RET51(Y1015F) kidneys was readily evident and included disorganization with irregular distribution of putative glomeruli throughout the renal parenchyma and duplicated kidneys (bar, 100 $\mu \mathrm{m})$. In contrast, wild-type (WT) and the other indicated RET mutant mice exhibit a well-formed outer cortex and inner medulla (separated by the dashed line) (bar, $200 \mu \mathrm{m})$. (B) Whole-mount DBA-FITC staining of E15.5 kidneys. (Top) Normal honeycomb pattern of UB branching, single ureters entering the bladder (white dot), and separation of wolffian duct (wd) from the ureter was evident in kidneys $(\mathrm{K})$ of mice expressing wild-type, RET51, and RET51(Y1062F). In RET51(Y1015F) animals, note bilateral small kidneys with supernumerary UBs (arrows) that enter the mesenchyme but show decreased branching and failed to separate from the wolffian duct (wd) (white dashed line). The testes (T) were also abnormally positioned. (Bottom) Imaging of the above kidneys by confocal microscopy clearly depicts the dramatic deficit in branching morphogenesis (arrowheads indicate branch points) in homozygous RET51(Y1015F) kidneys. Bar, $100 \mu \mathrm{m} .(C)$ Quantitation of the branching morphogenesis defect in E15.5 kidneys from the indicated wild-type and RET mutant mice $(n=3$ animals of each genotype, mean \pm SEM; [*] $P<0.05)$. (D) Branching defects in homozygous RET51(Y1015F) mice are kidney-autonomous. Embryonic

A

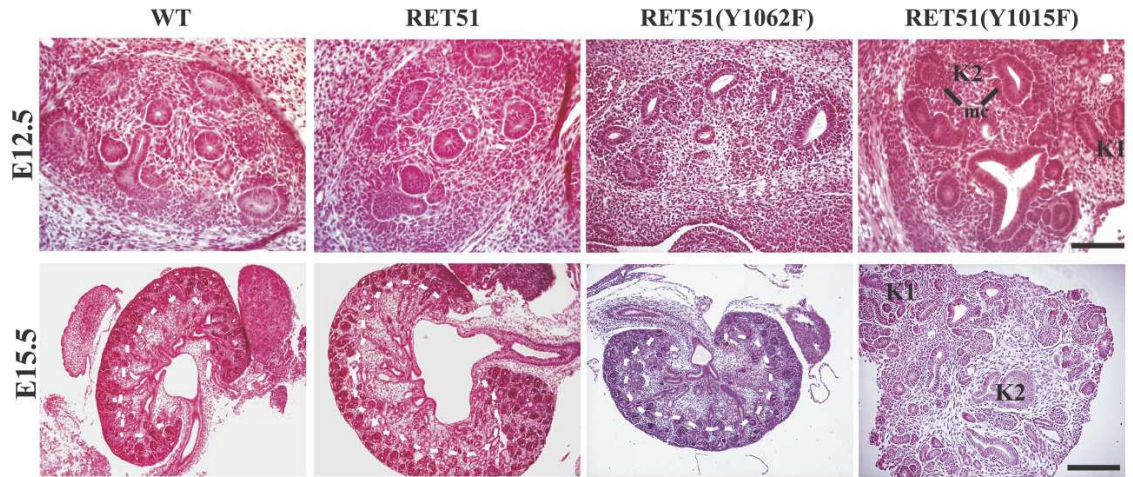

B
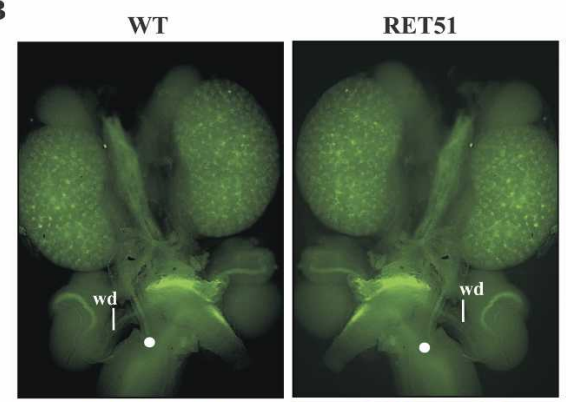

RET51(Y1062F)
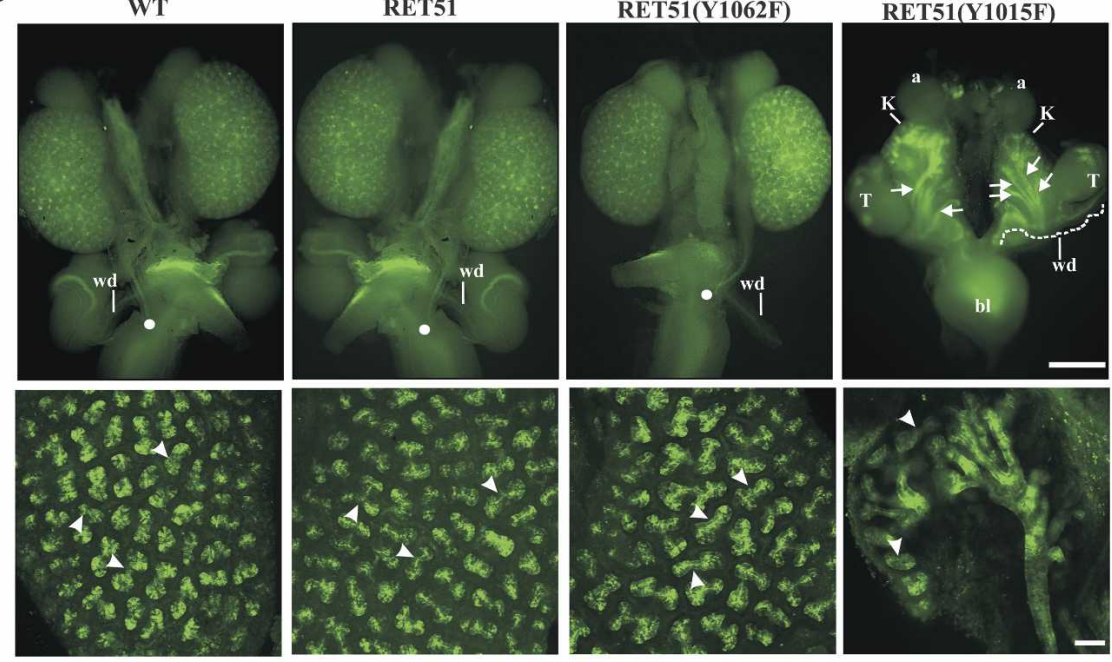

C

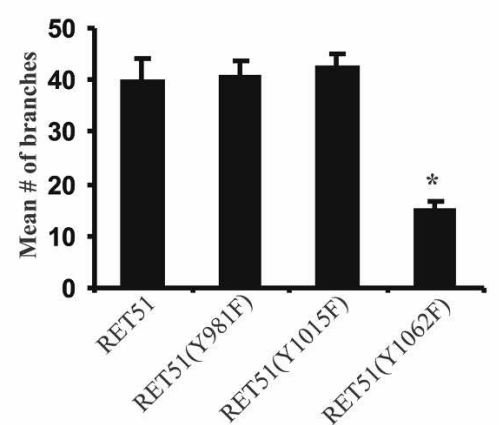

D

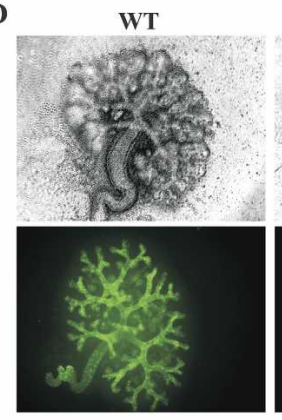

RET51(Y1015F)

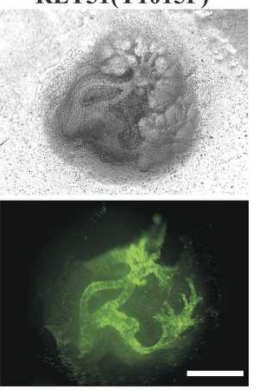

kidneys (E12.5) were dissected from the wolffian duct $(\mathrm{wd})$ and maintained in explant cultures. Visualization of the urinary tree in kidneys from RET51(Y1015F) mice shows abnormal branching (top row, phase contrast; bottom row, DBA-FITC staining). Bar, 75 um.

Cheng et al. 2003). If these processes were normal in Ret $^{\text {RET51(Y1O15F)/RET51(Y1015F) }}$ kidneys, one would expect (1) a punctate DBA staining pattern in the kidneys due to staining of branching UBs, (2) single ureters entering the bladder, and (3) separation of gonads and wolffian duct from the ureters as observed in wild-type and homozygous RET51 and RET51(Y1062F) animals (Fig. 4B). Instead, DBA staining of the Ret ${ }^{\text {RET51(Y1O15F)/RET51(Y1O15F) }}$ kidneys demonstrates bilateral supernumerary UBs that are attached to the wolffian duct, a severe decrease in branching morphogenesis, and failure of gonads to sepa- rate from the kidneys/ureters (Fig. 4B,C; data not shown). The presence of supernumerary UBs indicates that multiple UB inductions have occurred in these mutant mice. The decreased number of glomeruli is likely due to the reduced UB branching. The inability of the ureters to separate from the wolffian duct probably accounts for the distal obstruction seen in the ureters of postnatal mice as it likely poses an obstruction to urine outflow. This would explain the histopathological features of obstructive nephropathy observed in the postnatal kidneys of mutant mice. 
Reduced branching morphogenesis is

kidney-autonomous in $\operatorname{Ret}^{\text {RET51(Y1015F)/RET51(Y1015F) mice }}$

The developing murine kidney starts urine production after E13.5 (Vize et al. 2003). Therefore, it is possible that the decreased branching morphogenesis and the decreased number of glomeruli were due to obstruction and not due to inherent defects in UB branching. To eliminate this possibility, we cultured E12.5 wild-type and Ret $^{\text {RET51(Y1O15F)/RET51(Y1O15F) kidneys (dissected away }}$ from the wolffian ducts) in vitro, and visualized UB branching morphogenesis by DBA staining. In this setting, the wild-type kidneys demonstrate normal UB branching pattern, but the Ret ${ }^{\text {RET51(Y1015F)/RET51(Y1O15F) }}$ kidneys exhibit multiple ureters in the mesenchyme with abortive attempts to branch (Fig. 4D). From the above results, we conclude that RET51(Y1015) signaling is necessary for ensuring outgrowth of a single UB and promoting branching morphogenesis in the developing kidney.

Decreased PLC activation and increased apoptosis result in branching morphogenesis abnormalities in $\operatorname{Ret}^{\mathrm{RET} 51(\mathrm{Y} 1015 \mathrm{~F}) / \mathrm{RET} 51(\mathrm{Y} 1015 \mathrm{~F})}$ kidneys

Studies on biochemical characterization of oncogenic RET signaling mutants in cell culture suggest that RET(Y1015) and RET(Y1062) are key docking tyrosines that activate PLC $\gamma$ and AKT/MAPK signaling cascades, respectively. We performed a series of experiments to confirm that PLC $\gamma$ and not AKT/MAPK is a target of RET51(Y1015) in vivo, and to glean further insights into the pathways and cellular processes that may explain the $\operatorname{Ret}^{\text {RET51(Y1O15F)/RET51(Y1O15F) }}$ phenotype. First, biochemical analyses was performed on extracts from SCG neurons of Ret RET51(Y1O15F)/RET51(Y1O15F) mice. These neurons were selected for biochemical analysis as they are GDNF responsive and develop normally in these mice (data not shown). Our results demonstrate that PLC $\gamma$ is not activated in homozygous RET51(Y1015F) mice, whereas wild-type and Ret RET51/RET51 mice show normal PLC $\gamma$ activation (Fig. 5A). Second, there was no difference between AKT and MAPK activation in $\operatorname{Ret}^{\text {RET51(Y1O15F)/RET51(Y1O15F) }}$ and Ret RET51/RET51 mice, supporting the view that the RET51(Y1015F) phenotype results from abrogation of PLC $\gamma$ but not AKT/MAPK signaling (Fig. 5B).

Third, as an alternative means to demonstrate that PLC-mediated signaling regulates renal branching, we treated E12.5 wild-type kidney explant cultures with a PLC-specific inhibitor, Edelfosine (Horowitz et al. 2005). We monitored renal branching with DBA staining and found that Edelfosine treatment resulted in a dose-dependent decrease in branching morphogenesis (Fig. 5C). Fourth, in other systems Edelfosine-mediated reduction in PLC $\gamma$ signaling induces apoptosis (Cabaner et al. 1999; Harguindey et al. 2000; Gajate and Mollinedo 2002). This fact raised the possibility that decreased renal branching and nephrogenesis in homozygous RET51(Y1015F) mice could be a result of increased apoptosis due to abroga-
A
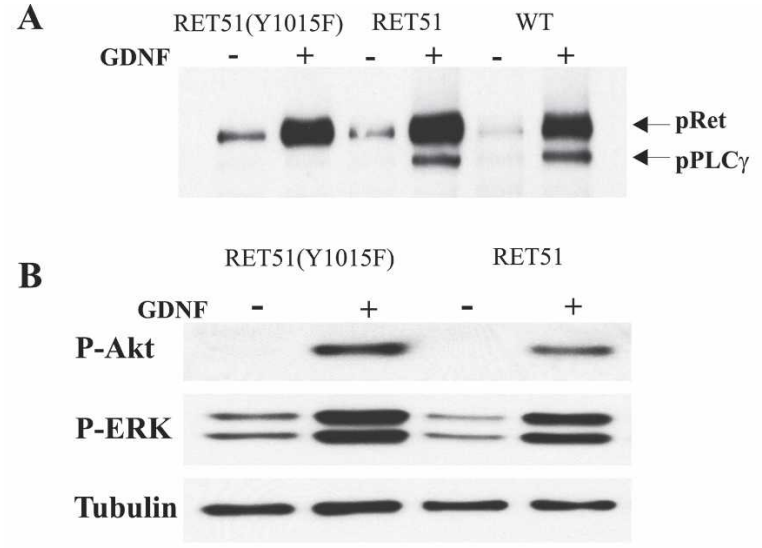

C

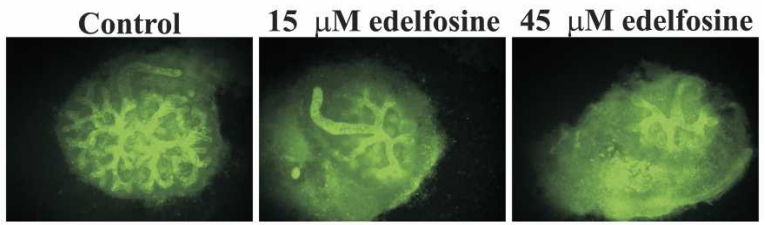

D
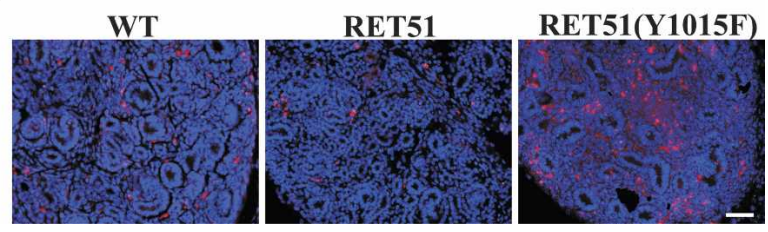

Figure 5. Kidney deficits in RET51(Y1015F) mice result from abnormalities in PLC $\gamma$ signaling and increased apoptosis. $(A)$ Western blots of primary SCG neuron lysates immunoblotted (IB) with phospho PLC $\gamma$ (pPLC $\gamma$ ) show absence of GDNF-stimulated pPLC $\gamma$ in homozygous RET51(Y1015F) compared with control lysates (homozygous RET51 and wild-type animals). Note that the antibody cross-reacts with phosphorylated RET that serves as an internal control, indicating no overt effect on total RET phosphorylation (see Materials and Methods). (B) Similar experiment as above with pAKT and pERK antibodies shows that the activation of AKT and MAPK is normal in RET51(Y1015F) animals. (C) PLC inhibition in wild-type kidneys recapitulates decreased renal branching phenotype. A PIPLC inhibitor, Edelfosine, shows a dose-dependent decrease in UB branching relative to control (vehicle-treated) E12.5 wildtype (WT) kidney explants (visualized with DBA-FITC; $n>5$ ). (D) The kidneys of homozygous RET51(Y1015F) mice show increased apoptosis as depicted by TUNEL staining (red nuclei), compared with wild-type and homozygous RET51 E15.5 kidneys ( $n \geq 3$ animals). Bar, $50 \mu \mathrm{m}$.

tion of PLC $\gamma$ signaling in these mice. Indeed, when compared with wild-type and Ret RET51/RET51 kidneys, $\operatorname{Ret}^{\text {RET51(Y1O15F)/RET51(Y1015F) }}$ kidneys show a dramatic increase in apoptosis as assessed by TUNEL staining (Fig. 5D). These results, when taken together along with kidney defects observed in PLC $\gamma 1$-null chimeras, provide compelling evidence that abrogation of RET51(Y1015)mediated PLC $\gamma 1$ signaling and resultant apoptosis are responsible for defective branching morphogenesis in the 
$\operatorname{Ret}^{\text {RET51(Y1015F)/RET51(Y1O15F) }}$ kidneys (Shirane et al. 2001), and suggest that patients with CAKUT may also have PLC signaling abnormalities.

\section{Roles of RET docking tyrosines in the context of RET9 isoform}

The above studies elucidated the physiologic roles of signaling pathways emanating from the major phosphotyrosine residues in the context of the RET51 isoform. To determine whether abrogation of signals from these residues in the RET9 isoform also resulted in physiologic deficits, we characterized renal development in mice with mutations in these docking tyrosines in the RET9 isoform (Y981, Y1015, and Y1062). Morphological analysis revealed partially penetrant unilateral megaureter in

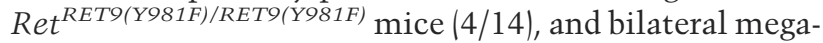
ureter, multicystic, and hypodysplastic kidneys in all

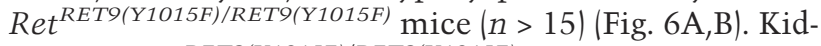
neys of $\operatorname{Ret}^{\text {RET9(Y1O15F)/RET9(Y1O15F) }}$ mice displayed features similar to those of $\operatorname{Ret}^{\text {RET51(Y1O15F)/RET51(Y1O15F) }}$ mice including supernumerary UBs, hypodysplastic kidneys, and failure of gonads to separate from wolffian ducts (Fig. 6A-C). This suggests that in kidney development, signals emanating from Y1015 in both RET9 and RET51 are equivalent.

More interestingly, in contrast to apparently normal kidneys of homozygous RET51(Y1062F) mice, the kidneys of all Ret ${ }^{R E T 9(Y 1062 F) / R E T 9(Y 1 O 62 F)}$ mice were abnormal (100\% penetrance) and displayed variable expressivity including bilateral agenesis, unilateral agenesis, and kidney rudiments (Fig. 6A-C). The histological abnormalities observed in the rudiments are similar to Retnull mice and include markedly reduced numbers of glomeruli, dysplasia, multiple cysts, and absence of nephrogenic zone $(n>5)$. The ureters and gonads were normal. Thus, compared with kidneys of homozygous RET51(Y1062F) mice, kidney development was severely affected in homozygous RET9(Y0162F) mice. It should be noted that numerous studies have shown that the Y1062F mutation does not affect RET stability or overall phosphorylation; thus the deficits in RET9(Y0162F) mice are likely to result from aberrant RET signaling through this critical docking tyrosine (Asai et al. 1996; SegouffinCariou and Billaud 2000; Jain et al. 2004; Jijiwa et al. 2004). The similarity of the kidney phenotype of these mice to Ret nulls suggests that signaling cascades activated by Y1062 are critical for initiating UB outgrowth.

$\mathrm{RET}(\mathrm{Y} 1062)$ is a critical tyrosine for activation of AKT and MAPK signaling cascades, yet in our studies we saw a major kidney defect only when the Y1062F mutation was present in RET9 (Besset et al. 2000; Hayashi et al. 2000). One reason that activation of AKT and MAPK is relatively normal in $\operatorname{Ret}^{\text {RET51(Y1O62F)/RET51(Y1O62F) }}$ kidneys is because these signaling pathways in RET51 can also be activated by RET(Y1096), which is not present in RET9 (Besset et al. 2000). Consistent with this reasoning, comparison of AKT and MAPK activation in Ret $^{\text {RET9(Y1062F)/RET9(Y1062F) }}$, Ret $^{\text {RET51(Y1O62F)/RET51(Y1062F) }}$ mice with the wild-type $\operatorname{Ret}^{R E T 9 / R E T 9}$ and $\operatorname{Ret}^{\text {RET51/RET51 }}$ mice, respectively, shows a marked decrease in AKT and MAPK phosphorylation in $\operatorname{Ret}^{\text {RET9(Y1062F)/RET9(Y1O62F) }}$ but not in Ret RET51(Y1062F)/RET51(Y1O62F) mice (Fig. 6D). To further support the idea that the C terminus of RET51 provides redundancy in kidney development, we generated hybrid-isoform mice $\operatorname{Ret}^{\text {RET9(Y1O62F)/RET51(Y1O62F), }}$ which harbor the Y1062F mutation in each of the RET9 and RET51 alleles. These mice did not have any major renal anomalies $(n=3)$ (Fig. 6B). Normal activation of AKT and MAPK in homozygous RET51(Y1062F) animals due to redundancy may explain the normal kidney development in homozygous RET51(Y1062F) mice.

\section{Discussion}

Loss-of-function studies have demonstrated essential roles of several proteins in kidney development and congenital renal disease including GDNF, GFR $\alpha 1, \mathrm{RET}$, Wnt11, SPRY1, SLIT2/ROBO2, WT1, and PAX2 (Yu et al. 2004). However, what components or signaling pathways lead to the abnormal phenotypes is unclear. To identify the signaling pathways mediated by RET that are important in GU development, we generated several "humanized" mutant RET mice that disrupt key RETdependent signal transduction pathways. The results provide several novel insights into RET biology, RTK signaling specificity, kidney development, and molecular pathogenesis of CAKUT and provide animal models of congenital renal diseases.

First, we discovered that signaling by either RET9 or RET51 is sufficient for kidney formation, that is, the wild-type human RET isoforms have redundant roles in GU development, and the human RET forms can function in mice. This result was surprising since a previous study on chimeric mouse-human Ret monoisomorphic mice found that the chimeric Ret51 did not support normal kidney development and resulted in perinatal lethality (de Graaff et al. 2001). Using RNA analysis, we excluded the possibility that our RET51 homozygous mice expressed any endogenous mouse Ret or Ret9 mRNA that could account for our different results. The levels of RET and ligand-dependent activation of AKT and MAPK, a critical pathway for RET activity, were also similar in our RET monoisomorphic mice. Importantly, four other RET51 knockin mice with different mutations [RET51(Y981F), RET51(Y1062F), RET51(Cdel), and RET9(51C)] also had essentially normal kidney development, indicating that the human RET51 can clearly sustain kidney development in mice. Differences between our work and the study of de Graaff et al. (2001) may be attributed to factors such as environmental or genetic strain differences or the different properties of human RET51 (used in our study) and chimeric murine Ret51 (used in the de Graaff study). Support for this latter idea consistent with our findings comes from the observation that wild-type human RET51 and RET9 promote branching morphogenesis to a similar extent, but the Y1062F mutation abrogates this activity only in RET9 and not in RET51, presumably because of redundancy through Y1096 (see below) (Degl'Innocenti et al. 2004). In con- 
A

B
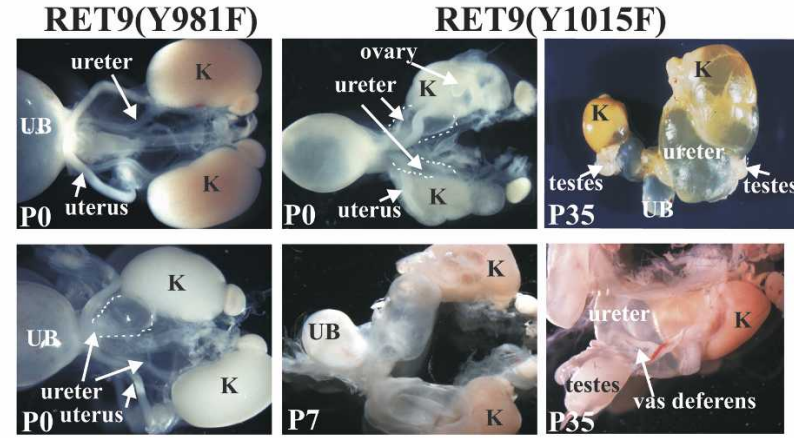

RET9(Y981F)/

RET9(Y981F)
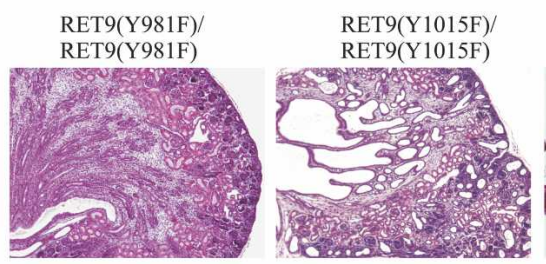

RET9(Y1062F)
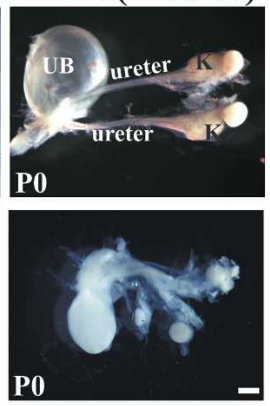

P0

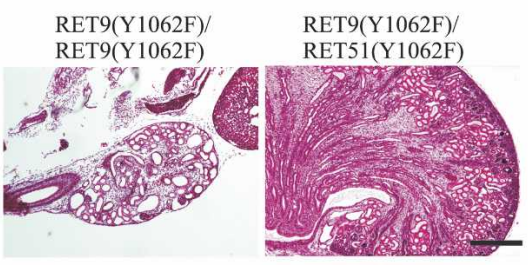

C
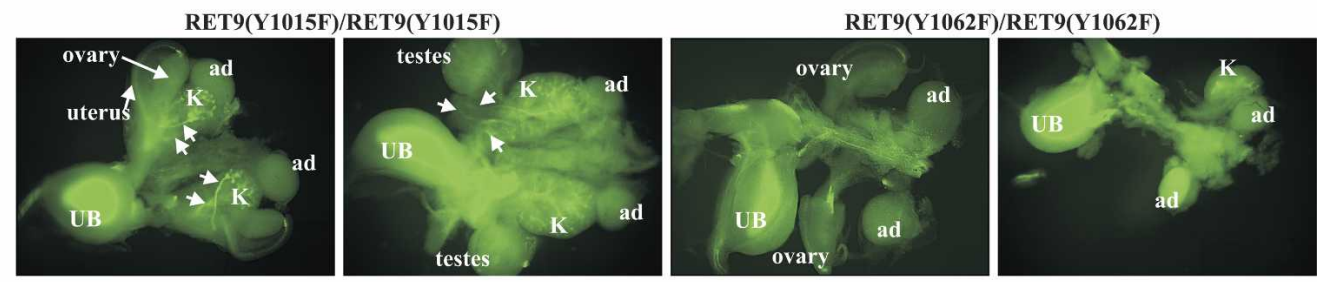

D
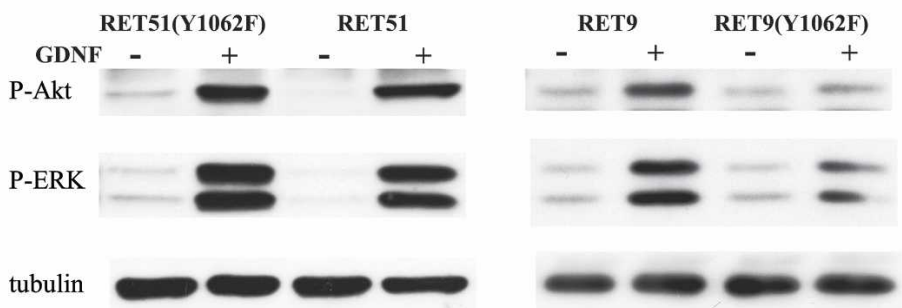

Figure 6. GU development in mice expressing docking tyrosine mutations in the RET9 isoform. (A) Gross depiction of spectrum of renal anomalies at the indicated ages in mice expressing RET9(Y981F), RET9(Y1015F), and RET9(Y1062F). In RET9(Y981F), partial penetrance of unilateral dilated ureter (dashed line, 4/12 animals) is shown. Examples of the progression of renal abnormalities in mice expressing RET9(Y1015F) show dilated ureters and abnormally positioned gonads at P0. By postnatal day 7 (P7), megaureters and compression of the kidneys were evident. By P35, normal kidney tissue was absent, and the testes and the vas deferens were attached to the proximal portion of the dilated ureter. In mice expressing RET9(Y1062F), renal agenesis (unilateral and bilateral) or kidney rudiments (cf. the size of the kidney with adrenal glands) were observed. The ureters and gonads of these animals were grossly normal. (B) Renal dysplasia in mice expressing RET9(Y1015F) or RET9(Y1062F). H\&E-stained sections of the indicated kidneys at P0 depict the dysplastic appearance in kidneys of homozygous RET9(Y1015F) and RET9(Y1062F)mice. Note that the presence of RET51(Y1062F) in the heteroisomorphic RET(Y1062F) kidneys (Ret ${ }^{\text {RET9(Y1062F)/RET51(Y1062F) }}$ ) resulted in histologically normal kidneys, indicating the importance of redundant signaling emanating from the RET51 C terminus in kidney development. (C) Whole-mount DBA-FITC staining of E15.5 homozygous RET9(Y1015F) and RET9(Y1062F) kidneys reveals that the defects in mice expressing RET9(Y1015F) (supernumerary UBs, decreased branching, abnormal gonads) were similar to kidney defects in mice expressing RET51(Y1015F). At E15.5, renal agenesis or rudiments were evident in mice expressing RET9(Y1062F) as opposed to normal development of kidneys of mice expressing RET51(Y1062F) (see Fig. 4). (D) Comparison of AKT and MAPK activation in homozygous RET9(Y1062F) and RET51(Y1062F) mice. Western blots of SCG lysates (as in Fig. 5) from mice expressing the indicated RET mutations show that AKT and MAPK activation is severely decreased in mice expressing RET9(Y1062F) compared with those expressing RET9, RET51, or RET51(Y1062F). Bars, $A, 2 \mathrm{~mm} ; B, 200 \mu \mathrm{m}$.

trast, murine Ret9, and not Ret51, promotes tubulogenesis (Schuetz et al. 2004).

The only urinary phenotype we observed in wild-type monoisoformic mice is partial penetrance for unilateral dilated ureters in a small number of homozygous RET9 $(3 / 50)$ and RET51 (1/50) mice. Some studies have suggested that human RET is less active than mouse Ret due to differences in their ability to interact with the 
GFLs and may explain the unilateral, partially penetrant GU defects in humanized monoisomorphic RET mice (Skinner et al. 2005). Because unilateral GU abnormalities are one of the commonest presentations of CAKUT, we suggest that some of this incidence may be due to decreased RET activity, as differences in RET signaling can result in incomplete penetrance and variable expressivity (Moore et al. 1996; Pichel et al. 1996; Sanchez et al. 1996; McCallion et al. 2003; Jain et al. 2004; Jijiwa et al. 2004).

Second, we discovered that the docking tyrosines of both RET9 and RET51 have distinct as well as redundant physiological roles in kidney development. Our biochemical, embryological, genetic, and pharmacological analyses of the GU system of homozygous RET(Y1015F) mice clearly establish the importance of RET(Y1015)mediated PLC $\gamma 1$ activation in ensuring proper outgrowth of a single UB and branching morphogenesis. Disruption of PLC $\gamma 1$ signaling results in supernumerary UBs, branching morphogenesis defects, hypodysplastic kidneys, failure of gonads to separate from the urinary tract, and multiple cysts ultimately leading to end-stage kidneys by 1 mo after birth. In these mice, the UBs are capable of invading the metanephric mesenchyme and initiating early branching, indicating that RET(Y1015) signaling is not required for these events. Rather, the UB outgrowth and early branching are regulated by RET9(Y1062) since homozygous RET9(Y1062F) mice either do not develop kidneys or have only kidney rudiments, similar to Ret-null animals (see below). While the role of PLC $\gamma 1$ in renal development was previously suggested from analysis of high percentage chimeras generated from $p l c \gamma 1^{-1-}$ ES cells (Plc $\gamma 1$-null mice are embryonically lethal), our results now provide evidence that this requirement is largely, if not entirely, mediated by RET(Y1015) (Shirane et al. 2001).

The fact that Y1015F kidneys do exhibit some branching, albeit decreased, suggests that other signaling pathways may also contribute to this event after initial UB induction. We could not evaluate this possibility in our monoisomorphic RET9(Y1062F) mice (because of renal agenesis), or in RET51(Y1062) mice since they had normal renal branching. However, diminished, but not absent, renal branching is observed in mice where signaling through endogenous mouse Ret(Y1062) is disrupted (Jijiwa et al. 2004). Together, these studies support the idea that normal branching requires intact signaling by both Y1015 and Y1062. Since proper branching morphogenesis prevents apoptosis of the mesenchyme and ensures normal nephron differentiation, defects in branching morphogenesis explain the increased apoptosis, hypodysplastic kidneys, and decreased glomeruli in RET(Y1015F) kidneys. Thus, different signaling pathways activated by RET (PLC $\gamma 1$ vs. AKT/MAPK) have distinct as well as redundant functions in kidney development. Whether Y981 has a role in kidney development is uncertain because of the partial penetrance of unilateral ureter defects in homozygous RET9 and RET9(Y981F). Our initial analysis revealed that the incidence of these partially penetrant GU defects is higher in homozygous RET9(Y981F) animals (4/12) compared with wild-type RET9 animals (3/50), suggesting that Y981 may only have a modest role, if any, in kidney development ( $p=0.03$, Fisher's exact test).

A third important insight from our studies is that the mechanism of Sproutyl regulation by Ret may be via RET(Y1015)-mediated PLC $\gamma 1$ activation (Basson et al. 2005). The GU phenotype of homozygous RET(Y1015F) is remarkably similar to Sprouty1-null mice (e.g., supernumerary UBs, failure of vas deferens to separate from the ureters, hypodysplastic kidneys, gonads attached to the GU system) and to other mouse models in which aberrant Sprouty expression affects UB branching and outgrowth by modulating Wnt11-GDNF-Ret signaling (Majumdar et al. 2003; Chi et al. 2004; Basson et al. 2005). These studies demonstrate that absence of Ret signaling results in loss of Sproutyl expression in wolffian ducts, which, in turn, renders these ducts more sensitive to GDNF and results in induction of supernumerary UBs. The phenotypic similarity between homozygous RET(Y1015F)- and Sprouty1-null mice, along with observations demonstrating that $R E T(Y 1015)$ is the major docking tyrosine for PLC $\gamma 1$ activation and that Sprouty expression is regulated by PLC $\gamma$ (Abe and Naski 2004), strongly suggest that Sprouty signaling may be modulated by RET(Y1015)-mediated PLC $\gamma$ activation.

Sproutyl proteins are repressors of RTK signaling and interact with various adaptor proteins (Kim and Bar-Sagi 2004). It is possible that RET51(Y1015F) mutation results in decreased Sprouty expression in wolffian ducts, or causes aberrant signaling through GRB2-SOS complexes due to failure of Sprouty phosphorylation. In addition, it was recently reported that deficient SLIT2 (expressed by UB tips)-mediated ROBO2 (expressed by mesenchyme) signaling results in supernumerary UBs and ureter remodeling abnormalities similar to those present in $\mathrm{RET}(\mathrm{Y} 1015 \mathrm{~F})$ mice and other mutant mice with aberrant GDNF/Ret signaling (Sainio et al. 1997; Batourina et al. 2002; Grieshammer et al. 2004). Whether Sprouty1 or SLIT2-ROBO2 is a component of the pathway regulated by RET(Y1015) will require further study.

The fourth important finding of our studies is the in vivo evidence that the $\mathrm{C}$ terminus of RET51, which harbors an additional docking site (Y1096) not present in RET9, provides redundancy in kidney development through activation of AKT/MAPK pathways. The residue Y1062 is present in both RET9 and RET51 and interacts with $\mathrm{PTB} / \mathrm{SH} 2$ domain-containing adaptor proteins including Shc, Doks $1-6$, and FRS1 to activate PI3K-AKT and ERK-MAPK pathways. Because we generated monoisoformic mice with impairment of signaling through Y1062, we were able to compare the role of this residue in the context of both isoforms. Our results demonstrate that kidneys do not develop or are rudimentary in RET9(Y1062F) animals. This phenotype is similar to that of Ret-null mice and to the recent report by Wong et al. (2005) that Ret9-Y1062F signaling is critical for UB outgrowth and enteric nervous system development. The markedly diminished to absent AKT/MAPK activation in these mice also shows that this pathway is im- 
portant for UB outgrowth (Schuchardt et al. 1996; Fisher et al. 2001; Tang et al. 2002). Such severe abnormalities are not present in RET51(Y1062F) mice because AKT/ MAPK activation is relatively intact in these mice, presumably due to redundancy from Y1096-Grb2 signaling as reported in other studies (Lorenzo et al. 1997; Besset et al. 2000; Degl'Innocenti et al. 2004).

An important observation from this study and others is that variable expressivity of renal abnormalities due to defects in UB outgrowth correlates with differences in RET-mediated AKT/MAPK activation (Jain et al. 2004; Jijiwa et al. 2004). For example, complete loss of signaling through RET(Y1062) as seen in RET9(Y1062F) or Retnull mice results in renal agenesis, while diminished RET-mediated AKT/MAPK activity, as occurs in RetDN mice, results in less severe renal abnormalities that include hypoplasia and incomplete penetrance of renal agenesis (Jain et al. 2004). Similarly, a mutation at Y1062 in the endogenous mouse Ret locus results in renal hypoplasia but not renal agenesis, presumably because Ret9 is more abundant than Ret51 in murine embryonic kidneys (Lee et al. 2003; Jijiwa et al. 2004). Finally, double heterozygotes containing a single allele of each of knocked-in human RET9(Y1062F) and RET51(Y1062F) have normal kidneys, again demonstrating the redundant signaling occurring via the RET51(Y1096) residue. All these studies taken together suggest that a wide spectrum of congenital anomalies due to UB outgrowth in humans can be due to alterations in the levels of the RET-dependent AKT/MAPK pathway.

Distinct biological activities of individual isoforms of a number of other RTKs (e.g., Met, PDGFR, insulin receptor, FGFR1) have been observed (Orian-Rousseau et al. 2002; Pandini et al. 2002). Our results in vivo present for the first time that key signaling pathways dependent on RET also have distinct and redundant roles in kidney development. Renal tract malformations account for $30 \%$ of anomalies diagnosed prenatally, and renal malformations are a major cause of chronic renal failure in children (Noia et al. 1989; Woolf and Thiruchelvam 2001). Our results from genetic experiments in mice phenocopy several of these anomalies and provide potentially important paradigms for understanding the molecular basis for these diseases.

\section{Materials and methods}

\section{Generation of various Ret knockin mice}

Institution-approved protocols were followed for all animal studies. Human RET9, RET51, and the indicated mutant cDNAs were homologously recombined into the first coding exon of the Ret gene to disrupt synthesis of the endogenous mouse Ret as previously described (Fig. 1A,B; Enomoto et al. 2001; see Supplemental Material for details).

\section{Tissue RNA analysis}

Total RNA was isolated from brain and analyzed by RT-PCR as previously described (Svaren et al. 2000). Total Ret transcripts (representing endogenous mouse and the inserted human se- quences) were identified as before (Jain et al. 2004). Primers to detect mouse Ret and total (mouse and human) Ret51 or Ret9 are described in the Supplemental Material.

\section{Histopathological analysis and immunohistochemistry}

Mouse tissues were fixed in 4\% paraformaldehyde and processed as previously described (Enomoto et al. 2000). Routine histological assessment was done on hematoxylin and eosinstained (H\&E) tissue sections. Cell death was analyzed using TUNEL assay (Boehringer Mannheim) (Enomoto et al. 2000). Immunohistochemistry with anti-RET antibody (Neuromics; 1: 100 , goat polyclonal) and visualization of the urinary tree with DBA is described in the Supplemental Material.

\section{Western blot analysis}

Western blots of cell extracts from primary SCG cultures were performed as described previously, and the details of the antibodies used are provided in the Supplemental Material (TsuiPierchala et al. 2002; Encinas et al. 2004).

\section{Embryonic kidney organ cultures and treatments}

Developing kidneys from E12.5 wild-type or RET mutant embryos were dissected away from wolffian duct and cultured at air-fluid interface as previously described (Supplemental Material; Rogers et al. 1991; Cheng et al. 2003). The organ cultures were maintained for $72 \mathrm{~h}$ and then fixed for $10 \mathrm{~min}$, washed in PBS, and stained with FITC-DBA for analysis. Conditions for treatment with the PLC inhibitor Edelfosine (Calbiochem) are described in the Supplemental Material.

\section{Microscopic analyses and quantitation}

Images for brightfield and immunofluorescence on tissue sections (Nikon Eclipse 80i), gross morphology (Nikon), and DBAFITC whole-mount specimens (Leica) were captured (CoolSnapES camera for fluorescence and CoolSnapCF for color images) and processed using Metamorph and Adobe Photoshop CS software. Visualization of the E15.5 kidneys at high resolution and quantitation of branching were done by confocal microscopy as detailed in the Supplemental Material. Glomerular counts were obtained from P0 kidneys that were sectioned in entirety as previously described (Jain et al. 2004). Quantitative analyses were performed with SigmaPlot software (SPSS Inc.), and statistical significance was determined with the Student $t$-test unless otherwise specified. For all studies, the sample size was three or more for each genotype.

\section{Acknowledgments}

We thank Kelli Simburger, Amber Nielson, Amy Strickland, Amanda Knoten, Tatiana Gorodinsky, and Nina Panchenko for excellent technical assistance. We are grateful to Dr. Marc Hammerman and Sharon Rogers for assistance with kidney explant cultures and Jason Gustin for numerous suggestions. This work was supported by the HOPE Center for Neurological Disorders; NIH grants AG13730 and NS39358 to J.M., AG13729 to E.M.J, and HD047396-01 to S.J.; and a grant from the Spanish "Ministerio de Educacion y Ciencia" BFU2004-03632 to M.E. M.E. holds a contract from the Spanish "Ramon y Cajal" program. 


\section{References}

Abe, M. and Naski, M.C. 2004. Regulation of sprouty expression by PLC $\gamma$ and calcium-dependent signals. Biochem. Biophys. Res. Commun. 323: 1040-1047.

Airaksinen, M.S. and Saarma, M. 2002. The GDNF family: Signalling, biological functions and therapeutic value. Nat. Rev. Neurosci. 3: 383-394.

Asai, N., Murakami, H., Iwashita, T., and Takahashi, M. 1996. A mutation at tyrosine 1062 in MEN2A-Ret and MEN2B-Ret impairs their transforming activity and association with shc adaptor proteins. J. Biol. Chem. 271: 17644-17649.

Baloh, R.H., Enomoto, H., Johnson Jr., E.M., and Milbrandt, J. 2000. The GDNF family ligands and receptors-Implications for neural development. Curr. Opin. Neurobiol. 10: $103-110$.

Basson, M.A., Akbulut, S., Watson-Johnson, J., Simon, R., Carroll, T.J., Shakya, R., Gross, I., Martin, G.R., Lufkin, T., McMahon, A.P., et al. 2005. Sproutyl is a critical regulator of GDNF/RET-mediated kidney induction. Dev. Cell 8: 229239.

Batourina, E., Choi, C., Paragas, N., Bello, N., Hensle, T., Costantini, F.D., Schuchardt, A., Bacallao, R.L., and Mendelsohn, C.L. 2002. Distal ureter morphogenesis depends on epithelial cell remodeling mediated by vitamin A and Ret. Nat. Genet. 32: 109-115.

Besset, V., Scott, R.P., and Ibanez, C.F. 2000. Signaling complexes and protein-protein interactions involved in the activation of the Ras and phosphatidylinositol 3-kinase pathways by the c-Ret receptor tyrosine kinase. J. Biol. Chem. 275: 39159-39166.

Borrello, M.G., Mercalli, E., Perego, C., Degl'Innocenti, D., Ghizzoni, S., Arighi, E., Eroini, B., Rizzetti, M.G., and Pierotti, M.A. 2002. Differential interaction of Enigma protein with the two RET isoforms. Biochem. Biophys. Res. Commun. 296: 515-522.

Cabaner, C., Gajate, C., Macho, A., Munoz, E., Modolell, M., and Mollinedo, F. 1999. Induction of apoptosis in human mitogen-activated peripheral blood T-lymphocytes by the ether phospholipid ET-18-OCH3: Involvement of the Fas receptor/ligand system. Br. J. Pharmacol. 127: 813-825.

Cacalano, G., Farinas, I., Wang, L.C., Hagler, K., Forgie, A., Moore, M., Armanini, M., Phillips, H., Ryan, A.M., Reichardt, L.F., et al. 1998. GFR $\alpha 1$ is an essential receptor component for GDNF in the developing nervous system and kidney. Neuron 21: 53-62.

Cheng, H.T., Miner, J.H., Lin, M., Tansey, M.G., Roth, K., and Kopan, R. 2003. $\gamma$-Secretase activity is dispensable for mesenchyme-to-epithelium transition but required for podocyte and proximal tubule formation in developing mouse kidney. Development 130: 5031-5042.

Chi, L., Zhang, S., Lin, Y., Prunskaite-Hyyrylainen, R., Vuolteenaho, R., Itaranta, P., and Vainio, S. 2004. Sprouty proteins regulate ureteric branching by coordinating reciprocal epithelial Wnt11, mesenchymal Gdnf and stromal Fgf7 signalling during kidney development. Development 131: 3345-3356.

Degl'Innocenti, D., Arighi, E., Popsueva, A., Sangregorio, R., Alberti, L., Rizzetti, M.G., Ferrario, C., Sariola, H., Pierotti, M.A., and Borrello, M.G. 2004. Differential requirement of Tyr1062 multidocking site by RET isoforms to promote neural cell scattering and epithelial cell branching. Oncogene 23: 7297-7309

de Graaff, E., Srinivas, S., Kilkenny, C., D'Agati, V., Mankoo, B.S., Costantini, F., and Pachnis, V. 2001. Differential activities of the RET tyrosine kinase receptor isoforms during mammalian embryogenesis. Genes \& Dev. 15: 2433-2444.

Encinas, M., Crowder, R.J., Milbrandt, J., and Johnson Jr., E.M. 2004. Tyrosine 981, a novel ret autophosphorylation site, binds c-Src to mediate neuronal survival. I. Biol. Chem. 279: 18262-18269.

Enomoto, H., Araki, T., Jackman, A., Heuckeroth, R.O., Snider, W.D., Johnson Jr., E.M., and Milbrandt, J. 1998. GFR $\alpha 1$ deficient mice have deficits in the enteric nervous system and kidneys. Neuron 21: 317-324.

Enomoto, H., Heuckeroth, R.O., Golden, J.P., Johnson, E.M., and Milbrandt, J. 2000. Development of cranial parasympathetic ganglia requires sequential actions of GDNF and neurturin. Development 127: 4877-4889.

Enomoto, H., Crawford, P.A., Gorodinsky, A., Heuckeroth, R.O., Johnson Jr., E.M., and Milbrandt, J. 2001. RET signaling is essential for migration, axonal growth and axon guidance of developing sympathetic neurons. Development 128: 3963-3974.

Fisher, C.E., Michael, L., Barnett, M.W., and Davies, J.A. 2001. Erk MAP kinase regulates branching morphogenesis in the developing mouse kidney. Development 128: 4329-4338.

Gajate, C. and Mollinedo, F. 2002. Biological activities, mechanisms of action and biomedical prospect of the antitumor ether phospholipid ET-18-OCH(3) (edelfosine), a proapoptotic agent in tumor cells. Curr. Drug Metab. 3: 491-525.

Grieshammer, U., Le, M., Plump, A.S., Wang, F., Tessier-Lavigne, M., and Martin, G.R. 2004. SLIT2-mediated ROBO2 signaling restricts kidney induction to a single site. Dev. Cell 6: 709-717.

Grisaru, S., Cano-Gauci, D., Tee, J., Filmus, J., and Rosenblum, N.D. 2001. Glypican-3 modulates BMP- and FGF-mediated effects during renal branching morphogenesis. Dev. Biol. 231: 31-46.

Harguindey, S., Pedraz, J.L., Canero, R.G., and Katin, M. 2000. Edelfosine, apoptosis, MDR and $\mathrm{Na}^{+} / \mathrm{H}^{+}$exchanger: Induction mechanisms and treatment implications. Apoptosis 5: $87-89$.

Hayashi, H., Ichihara, M., Iwashita, T., Murakami, H., Shimono, Y., Kawai, K., Kurokawa, K., Murakumo, Y., Imai, T., Funahashi, H., et al. 2000. Characterization of intracellular signals via tyrosine 1062 in RET activated by glial cell linederived neurotrophic factor. Oncogene 19: 4469-4475.

Horowitz, L.F., Hirdes, W., Suh, B.-C., Hilgemann, D.W., Mackie, K., and Hille, B. 2005. Phospholipase C in living cells: Activation, inhibition, $\mathrm{Ca}^{2+}$ requirement, and regulation of M current. J. Gen. Physiol. 126: 243-262.

Jain, S., Naughton, C.K., Yang, M., Strickland, A., Vij, K., Encinas, M., Golden, J., Heuckeroth, R., Johnson, E., and Milbrandt, J. 2004. Mice expressing a dominant-negative Ret mutation phenocopy human Hirschsprung disease and delineate a direct role of Ret in spermatogenesis. Development 131: 5503-5513.

Jijiwa, M., Fukuda, T., Kawai, K., Nakamura, A., Kurokawa, K., Murakumo, Y., Ichihara, M., and Takahashi, M. 2004. A targeting mutation of tyrosine 1062 in Ret causes a marked decrease of enteric neurons and renal hypoplasia. Mol. Cell. Biol. 24: 8026-8036.

Kim, H.J. and Bar-Sagi, D. 2004. Modulation of signalling by Sprouty: A developing story. Nat. Rev. Mol. Cell Biol. 5: 441-450.

Lechner, M.S. and Dressler, G.R. 1997. The molecular basis of embryonic kidney development. Mech. Dev. 62: 105-120.

Lee, K.Y., Samy, E.T., Sham, M.H., Tam, P.K., and Lui, V.C. 2003. 3' splicing variants of ret receptor tyrosine kinase are differentially expressed in mouse embryos and in adult mice. Biochim. Biophys. Acta 1627: 26-38. 
Liu, X., Vega, Q.C., Decker, R.A., Pandey, A., Worby, C.A., and Dixon, J.E. 1996. Oncogenic RET receptors display different autophosphorylation sites and substrate binding specificities. J. Biol. Chem. 271: 5309-5312.

Lorenzo, M.J., Gish, G.D., Houghton, C., Stonehouse, T.J., Pawson, T., Ponder, B.A., and Smith, D.P. 1997. RET alternate splicing influences the interaction of activated RET with the SH2 and PTB domains of Shc, and the SH2 domain of Grb2. Oncogene 14: 763-771.

Madhani, H.D. 2001. Accounting for specificity in receptor tyrosine kinase signaling. Cell 106: 9-11.

Majumdar, A., Vainio, S., Kispert, A., McMahon, J., and McMahon, A.P. 2003. Wnt11 and Ret/Gdnf pathways cooperate in regulating ureteric branching during metanephric kidney development. Development 130: 3175-3185.

McCallion, A.S., Stames, E., Conlon, R.A., and Chakravarti, A. 2003. Phenotype variation in two-locus mouse models of Hirschsprung disease: Tissue-specific interaction between Ret and Ednrb. Proc. Natl. Acad. Sci. 100: 1826-1831.

Moore, M.W., Klein, R.D., Farinas, I., Sauer, H., Armanini, M., Phillips, H., Reichart, L.F., Ryan, A.M., Carver-Moore, K., and Rosenthal, A. 1996. Renal and neuronal abnormalities in mice lacking GDNF. Nature 382: 76-79.

Noia, G., Masini, L., Caruso, A., Perrelli, L., Calisti, A., Salvaggio, E., and Mancuso, S. 1989. Prenatal diagnosis of congenital uropathies. Fetal Ther. 4 Suppl 1: 40-51.

Orian-Rousseau, V., Chen, L., Sleeman, J.P., Herrlich, P., and Ponta, H. 2002. CD44 is required for two consecutive steps in HGF/c-Met signaling. Genes \& Dev. 16: 3074-3086.

Pandini, G., Frasca, F., Mineo, R., Sciacca, L., Vigneri, R., and Belfiore, A. 2002. Insulin/insulin-like growth factor I hybrid receptors have different biological characteristics depending on the insulin receptor isoform involved. J. Biol. Chem. 277: 39684-39695.

Pichel, J.G., Shen, L., Hui, S.Z., Granholm, A.-C., Drago, J., Grinberg, A., Lee, E.J., Huang, S.P., Saarma, M., Hoffer, B.J., et al. 1996. Defects in enteric innervation and kidney development in mice lacking GDNF. Nature 382: 73-76.

Pope, J.C.T., Brock III, J.W., Adams, M.C., Stephens, F.D., and Ichikawa, I. 1999. How they begin and how they end: Classic and new theories for the development and deterioration of congenital anomalies of the kidney and urinary tract, CAKUT. J. Am. Soc. Nephrol. 10: 2018-2028.

Rogers, S.A., Ryan, G., and Hammerman, M.R. 1991. Insulinlike growth factors I and II are produced in the metanephros and are required for growth and development in vitro. J. Cell Biol. 113: 1447-1453.

Rossel, M., Pasini, A., Chappuis, S., Geneste, O., Fournier, L., Schuffenecker, I., Takahashi, M., van Grunsven, L.A., Urdiales, J.L., Rudkin, B.B., et al. 1997. Distinct biological properties of two RET isoforms activated by MEN 2A and MEN 2B mutations. Oncogene 14: 265-275.

Sainio, K., Suvanto, P., Saarma, M., Arum $\alpha$ e, U., Lindahl, M., Davies, J.A., and Sariola, H. 1997. Glial cell-line derived neurotrophic factor is a morphogen for the ureteric bud epithelium. Development 124: 4077-4087.

Sanchez, M.P., Silos-Santiago, I., Frisen, J., He, B., Lira, S.A., and Barbacid, M. 1996. Renal agenesis and the absence of enteric neurons in mice lacking GDNF. Nature 382: 70-73.

Sariola, H. and Saarma, M. 2003. Novel functions and signalling pathways for GDNF. J. Cell Sci. 116 (Pt 19): 3855-3862.

Schuchardt, A., D'Agati, V., Larsson-Blomberg, L., Costantini, F., and Pachnis, V. 1994. Defects in the kidney and enteric nervous system of mice lacking the tyrosine kinase receptor Ret. Nature 367: 380-383.

Schuchardt, A., D'Agati, V., Pachnis, V., and Costantini, F.
1996. Renal agenesis and hypodysplasia in ret-k- mutant mice result from defects in ureteric bud development. Development 122: 1919-1929.

Schuetz, G., Rosario, M., Grimm, J., Boeckers, T.M., Gundelfinger, E.D., and Birchmeier, W. 2004. The neuronal scaffold protein Shank3 mediates signaling and biological function of the receptor tyrosine kinase Ret in epithelial cells. I. Cell Biol. 167: 945-952.

Segouffin-Cariou, C. and Billaud, M. 2000. Transforming ability of MEN2A-RET requires activation of the phosphatidylinositol 3-Kinase/AKT signaling pathway. I. Biol. Chem. 275: 3568-3576.

Shirane, M., Sawa, H., Kobayashi, Y., Nakano, T., Kitajima, K., Shinkai, Y., Nagashima, K., and Negishi, I. 2001. Deficiency of phospholipase C- $\gamma 1$ impairs renal development and hematopoiesis. Development 128: 5173-5180.

Skinner, M.A., Kalyanaraman, S., Safford, S.D., Heuckeroth, R.O., Tourtellotte, W., Goyeau, D., Goodfellow, P., Milbrandt, J.D., and Freemerman, A. 2005. A human yeast artificial chromosome containing the multiple endocrine neoplasia type $2 \mathrm{~B}$ Ret mutation does not induce medullary thyroid carcinoma but does support the growth of kidneys and partially rescues enteric nervous system development in Ret-deficient mice. Am. J. Pathol. 166: 265-274.

Srinivas, S., Wu, Z., Chen, C.M., D'Agati, V., and Costantini, F. 1999. Dominant effects of RET receptor misexpression and ligand-independent RET signaling on ureteric bud development. Development 126: 1375-1386.

Svaren, J., Ehrig, T., Abdulkadir, S.A., Ehrengruber, M.U., Watson, M.A., and Milbrandt, J. 2000. EGR1 target genes in prostate carcinoma cells identified by microarray analysis. $J$. Biol. Chem. 275: 38524-38531.

Takahashi, M. 2001. The GDNF/RET signaling pathway and human diseases. Cytokine Growth Factor Rev. 12: 361-373.

Tang, M.J., Cai, Y., Tsai, S.J., Wang, Y.K., and Dressler, G.R. 2002. Ureteric bud outgrowth in response to RET activation is mediated by phosphatidylinositol 3-kinase. Dev. Biol. 243: 128-136.

Tsui-Pierchala, B.A., Ahrens, R.C., Crowder, R.J., Milbrandt, J., and Johnson Jr., E.M. 2002. The long and short isoforms of Ret function as independent signaling complexes. J. Biol. Chem. 277: 34618-34625.

Vize, P.D., Woolf, A.S., and Bard, J.B.L. 2003. The kidney from normal development to congenital disease. Academic Press, London.

Wong, A., Bogni, S., Kotka, P., de Graaff, E., D'Agati, V., Costantini, F., and Pachnis, V. 2005. Phosphotyrosine 1062 is critical for the in vivo activity of the Ret9 receptor tyrosine kinase isoform. Mol. Cell. Biol. 24: 9661-9673.

Woolf, A.S. and Thiruchelvam, N. 2001. Congenital obstructive uropathy: Its origin and contribution to end-stage renal disease in children. Adv. Ren. Replace Ther. 8: 157-163.

Yu, J., McMahon, A.P., and Valerius, M.T. 2004. Recent genetic studies of mouse kidney development. Curr. Opin. Genet. Dev. 14: 550-557. 


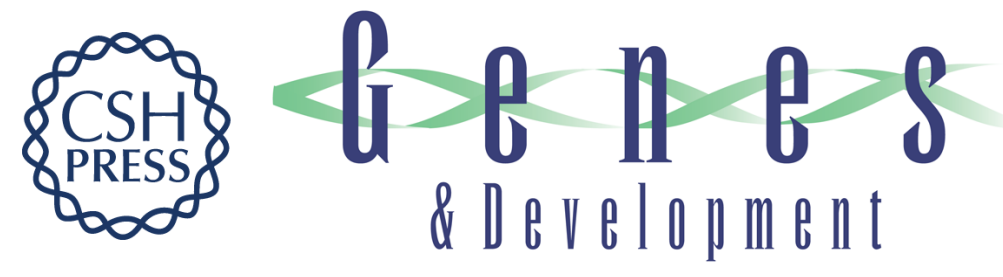

\section{Critical and distinct roles for key RET tyrosine docking sites in renal development}

Sanjay Jain, Mario Encinas, Eugene M. Johnson, Jr., et al.

Genes Dev. 2006, 20:

Access the most recent version at doi:10.1101/gad.1387206

\section{Supplemental http://genesdev.cshlp.org/content/suppl/2006/01/18/20.3.321.DC1 Material}

References This article cites 56 articles, 26 of which can be accessed free at: http://genesdev.cshlp.org/content/20/3/321.full.html\#ref-list-1

\section{License}

Email Alerting

Receive free email alerts when new articles cite this article - sign up in the box at the top Service 